\title{
When viruses collide: hepatitis B virus reactivation after hepatitis C treatment
}

\author{
Ashwin Balagopal and Chloe L. Thio \\ Department of Medicine, Division of Infectious Diseases, Johns Hopkins University School of Medicine, Baltimore, Maryland, USA.
}

\begin{abstract}
Treatment for hepatitis $\mathrm{C}$ virus (HCV) with direct-acting antivirals (DAAs) in hepatitis B virus (HBV) coinfection can result in HBV reactivation. In this issue of the $J C l$, Cheng and colleagues explored the role of interferon signaling in the complex interaction between HBV and HCV using cell lines, mouse models, and samples from people with coinfection. Notably, HCV enhanced interferon signaling, as measured by interferon-stimulated gene (ISC) expression, and decreased HBV transcription and replication. Blockade of interferon signaling reversed the effects on HBV replication. Further, pharmacologic inhibition of HCV replication in vitro and in coinfected humanized mice also reduced interferon signaling and, correspondingly, increased HBV replication. Intriguingly, baseline serum levels of the ISC CXCL10 predicted HBV reactivation in a cohort of coinfected people taking DAAs. Determining how interferon signaling silences HBV transcription and whether serum CXCL10 predicts $\mathrm{HBV}$ reactivation in a clinical setting are questions that warrant further investigation.
\end{abstract}

\section{HBV/HCV coinfection}

Viral hepatitis kills over 1 million people annually worldwide, and the majority of these deaths are attributable to chronic infection from either hepatitis B virus (HBV) or hepatitis $\mathrm{C}$ virus (HCV) (1). Accordingly, the WHO developed a strategy to eliminate viral hepatitis in the more than 270 million people who have chronic hepatitis $\mathrm{B}(\mathrm{CHB})$ (2) and more than 70 million who have chronic hepatitis $\mathrm{C}$ (CHC).(3) Because of shared routes of transmission, the prevalence of $\mathrm{CHB}$ in persons with $\mathrm{CHC}$ ranges from $1.4 \%$ to $35 \%(4,5)$.

Both viruses infect the liver but have different life cycles in the hepatocytes that they infect. HBV is a DNA virus whose replication template, the covalently closed circular DNA (cccDNA), resides in the nucleus of infected hepatocytes indefinitely, even in those who have developed protective antibodies. The HBV genome, which is transcribed from cccDNA, is replicated by the viral polymerase. Current HBV therapeutics that inhibit this viral polymerase do not directly eliminate cccDNA. Thus, $\mathrm{HBV}$ eradication, meaning the elimination of cccDNA, is challenging to achieve currently with available therapeutics.

Despite the durability of cccDNA, there is evidence that cellular processes can decrease or silence cccDNA transcription $(6,7)$. The mechanisms of this silencing are not fully understood, but when inhibited, there is potential for HBV reactivation. In HBV monoinfection, reactivation classically occurs during immunosuppression from cancer chemotherapy or with use of biologics such as rituximab $(8,9)$. So, clearly, in some cases, the host immune response can suppress HBV replication.

HBV-HCV coinfection presents a unique situation where the two viruses may have complex interactions. It was first

Delated Article: p. 3205

Conflict of interest: The authors have declared that no conflict of interest exists.

Copyright: () 2020, American Society for Clinical Investigation.

Reference information: / Clin Invest. 2020;130(6):2823-2826. https://doi.org/10.1172/JCI137477.

noticed that the viral kinetics of HBV and $\mathrm{HCV}$ are often reciprocal; high-level HCV viremia is accompanied by low-level or even absent HBV viremia with continued presence of hepatitis B surface antigen (HBsAg). Less commonly, HBV may be the predominant virus (10-12). One hypothesis to explain these findings included interference in viral replication between the two viruses, but there have been conflicting data regarding whether both viruses could replicate in the same hepatocyte $(13,14)$. Another hypothesis was that HCV activated the innate immune response, which, in turn, suppressed HBV replication $(15,16)$. HBV is considered a stealth virus in that it evades the innate immune response without actively inhibiting immunity $(17,18)$. Thus, if a second agent were to activate innate immune responses, the collateral effect may be to suppress HBV replication. For example, one study demonstrated that acute HIV infection in people with CHB decreased HBV DNA levels through a mechanism that might involve innate immune activation (19).

\section{The era of direct-acting antivirals}

The nature of the duel between HBV and $\mathrm{HCV}$ was complicated by the advent of direct-acting antivirals (DAAs), which revolutionized HCV treatment. Over 95\% of people with $\mathrm{CHC}$ achieve a sustained virologic response with DAAs. Despite this remarkable progress, there are numerous reports of $\mathrm{HBV}$ reactivation after initiating DAAs in HBV-HCV-coinfected people (20-22). Although the total number of cases was initially limited, instances of hepatic failure from reactivation led the FDA to issue a black box warning to monitor for possible $\mathrm{HBV}$ reactivation in people with HCV coinfection who initiate DAA treatment. Since the first reports, larger observational studies have strengthened the association of $\mathrm{HBV}$ reactivation with DAA treatment (23). Thus, there appeared to be a strong connection between $\mathrm{HBV}$ 
A HBV monoinfection

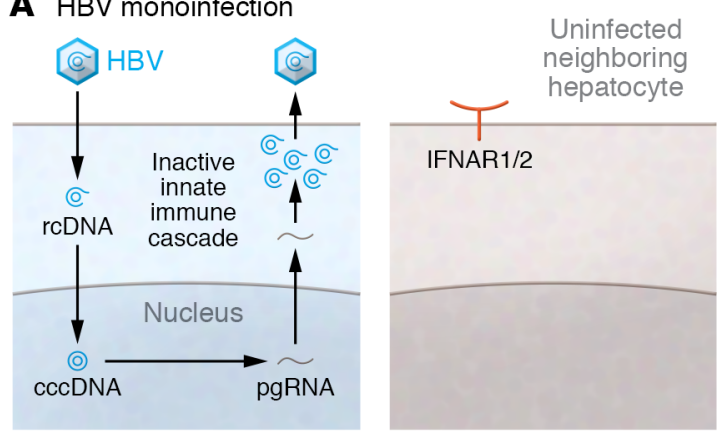

B HCV monoinfection

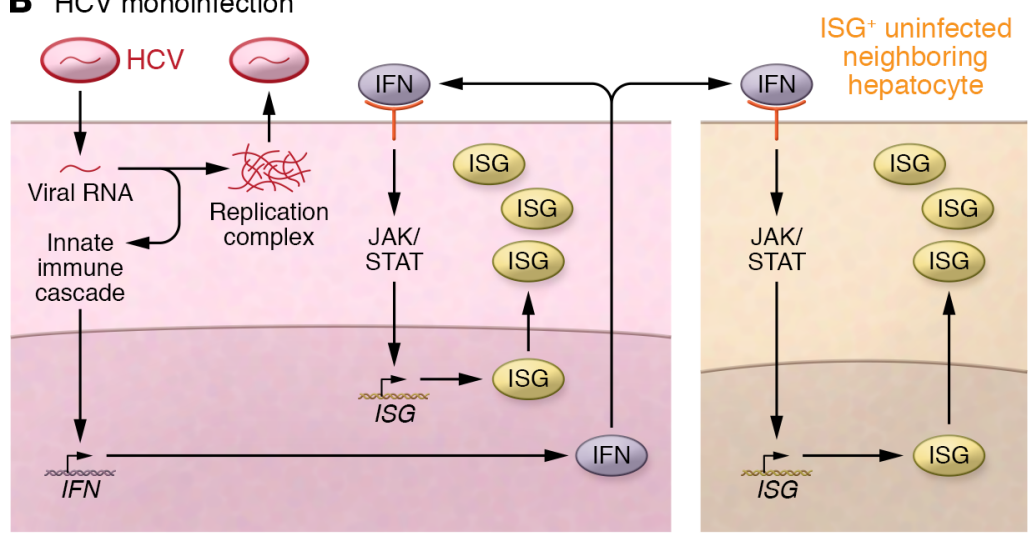

C HBV-HCV coinfection
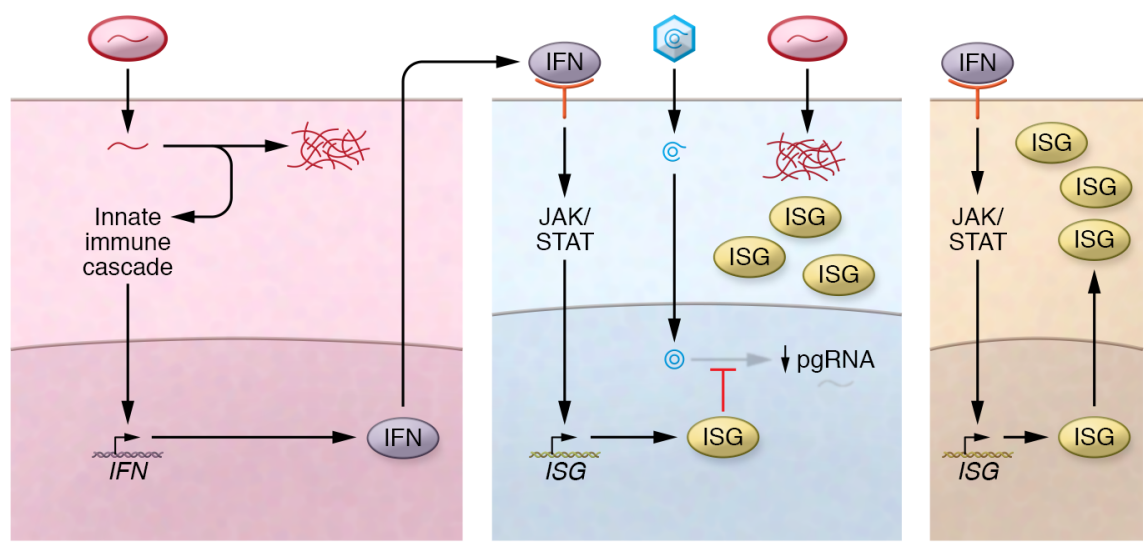

D HBV-HCV coinfection with DAAs or JAKi
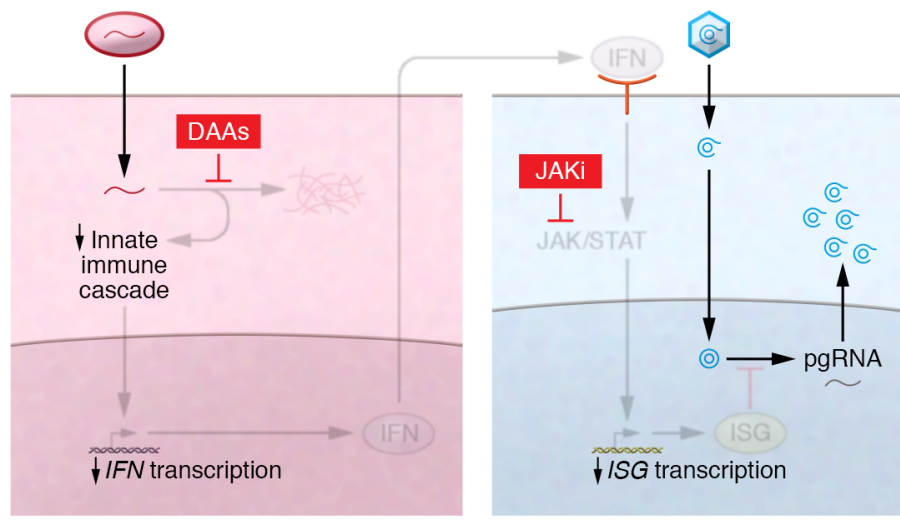

Figure 1. Hepatitis B and C coinfection are intertwined by the type 1 interferon response. (A) In HBV monoinfection, virions infect hepatocytes and uncoat, yielding the relaxed circular DNA (rcDNA) that is converted to covalently closed circular DNA (cccDNA) by host nuclear polymerases. cccDNA is the template for transcription of pregenomic RNA (pgRNA) and other viral RNAs (not pictured). pgRNA is exported into the cytoplasm, encapsidated, and reverse transcribed by the HBV polymerase into rcDNAs that egress as infectious virions. HBV fails to trigger the innate immune type 1 interferon signaling cascade. (B) In HCV monoinfection, virions infect hepatocytes and uncoat. The single-stranded RNA template is translated into nonstructural proteins that replicate progeny RNA molecules in membranous webs. Encapsidated progeny RNAs egress as infectious virions. The viral genomes trigger intracellular innate immune sensors, leading to type 1 interferon production. Type 1 interferons ligate interferon $\alpha$ receptors (IFNAR1/2) on neighboring hepatocytes, triggering JAK/STAT signaling that terminates in transcription of hundreds of antiviral ISGs. (c) In HBV-HCV coinfection, HCV infection triggers type 1 interferon release that is sensed by HBV-infected hepatocytes. Upregulated ISG expression in HBV-infected hepatocytes associates with decreased abundance of HBV transcripts and decreased HBV replication. (D) DAAs that inhibit HCV replication are associated with dampening type 1 interferon responses. Similarly, JAK inhibitors (Jaki) block the signaling cascade following IFNAR1/2 ligation, inhibiting upregulation of ISC expression. Both DAAs and JAKi result in the derepression of HBV transcription and increased HBV replication. This also increases the number of cells that show coinfection with $\mathrm{HBV}$ and $\mathrm{HCV}$.

and HCV without a clear mechanistic underpinning, although several groups postulated an immunologic link between the viruses.

\section{Interferon responses}

In the current issue of the JCI, Cheng et al. conducted a series of experiments involving cell culture, humanized mice, and samples taken from coinfected people that strongly implicate diminished interferon responses in $\mathrm{HBV}$ reactivation during DAA treatment in HBV-HCV coinfection (24). First, the investigators cocultured HBV and HCV by inoculating primary human hepatocytes with $\mathrm{HBV}$ followed five to six days later by adding HCV. In contrast to the control HBV monoinfection culture, levels of HBV DNA, HBsAg, hepatitis B e antigen (HBeAg), and HBV RNA decreased in the HBV-HCV coculture. Accompanying 
this decrease was an increase in expression of interferon-stimulated genes (ISGs), such as ISG15 and CXCL1O, both of which are upregulated during and due to $\mathrm{CHC}$. Taking these results together, the team hypothesized that HCV infection promotes innate immune activation vis-à-vis the potent interferon system, proposing that type 1 and 3 interferons are responsible for restricting HBV replication in coinfection. They demonstrated the link in HBV-HCV cocultures by showing that a broadly acting Janus kinase inhibitor (Jaki), which attenuates interferon responses by inhibiting downstream signaling, restored HBV titers in vitro. These data suggest that interferons exert control over HBV replication via transcriptional suppression of cccDNA because intracellular HBV RNA levels were repressed in HBV-HCV cocultures (24).

Cheng and colleagues validated these results using in situ methods for HCV RNA and HBV nucleic acid detection to confirm infection and to confirm that replication of both viruses occur in the same hepatocyte. In fact, the number of hepatocytes replicating both viruses increased from $12 \%$ to $58 \%$ with Jaki. Using this in situ system, they then modeled the clinical treatment scenario by adding sofosbuvir, a potent DAA, to HBV-HCV cocultures. As expected, by blocking $\mathrm{HCV}$ replication with sofosbuvir, ISG expression partially decreased. Intracellular HBV RNA levels increased to intermediate levels with the addition of sofosbuvir. In contrast, entecavir, a typical nucleoside analog (NUC) used for HBV, had no effect on innate immune signaling. These data support the idea that DAA treatment mediates $\mathrm{HBV}$ reactivation through a decrease in the interferon response.

Cheng et al. verified their findings using a longer-duration in vivo model: humanized mice that were infected with HBV and maintained for 10 weeks; approximately half of the animals were inoculated with $\mathrm{HCV}$ at week 4. HBV-HCVcoinfected animals had lower HBV titers than HBV-monoinfected mice, and correspondingly had upregulated hepatocyte ISG expression compared with monoinfected mice. DAA treatment of these animals suppressed HCV viremia and led to enhanced $\mathrm{HBV}$ viremia that corresponded with a decrease in serum CXCL10 levels. To validate the link between interferon responses and partial HBV control, the investigators measured serum CXCL10 levels in $35 \mathrm{HBV}-\mathrm{HCV}$-coinfected people who underwent DAA treatment and had characterized outcomes for HBV reactivation. Median baseline (pre-DAA) CXCL10 levels were higher in coinfected people who had HBV reactivation than in people who did not have reactivation. However, while baseline CXCL10 levels predicted which patients would reactivate $\mathrm{HBV}$, levels declined in most patients rapidly after DAA initiation, showing no differences between those who reactivated HBV and those who did not. This finding suggests that those with reactivation mounted a stronger interferon response to $\mathrm{HCV}$ and when that response was attenuated during DAA treatment, HBV reactivated. In those without $\mathrm{HBV}$ reactivation, a different mechanism was likely suppressing HBV replication. Cheng and authors concluded that HCV induces interferons that are responsible for suppression of $\mathrm{HBV}$, and that DAA-mediated clearance of $\mathrm{HCV}$ diminishes the stimulus for interferon secretion, thereby derepressing HBV transcription (ref. 24 and Figure 1).

\section{Further questions}

Cheng and colleagues should be lauded for undertaking this comprehensive mechanistic study to uncover a cause for $\mathrm{HBV}$ reactivation with DAA treatment in HCV-coinfected individuals (24). Although there are several parts of the study that raise further questions (would Janus kinase inhibition have led to increased HBV titers in humanized mice? Which ISGs are responsible for suppressing $\mathrm{HBV}$ replication? Is there chromatin remodeling of cccDNA that occurs after DAA initiation? Why is HCV replication occasionally suppressed in coinfection?), the methodical nature of the experiments conclusively supports the hypothesis that loss of the hepatic interferon response leads to some cases of $\mathrm{HBV}$ reactivation in HBV-HCV coinfection. The authors clearly demonstrate that both viruses can replicate in the same hepatocyte, so the "crowded replication space" hypothesis is unlikely to explain reciprocal viremia (24). However, their findings do not explain why only a minority of HBV-HCV-coinfected individuals who receive DAA treatment reactivate $\mathrm{HBV}$. It is likely that there are other mechanisms (immune or otherwise) that suppress HBV replication, and these may be more active in people with HBVHCV coinfection who do not reactivate. It is yet to be determined, however, how clinicians can exploit this new understanding of $\mathrm{HBV}$ reactivation to prevent its occurrence in people. Presently, in HBV-HCV-coinfected people who are not receiving NUCs, clinical monitoring for reactivation is the only guidance for those initiating DAA treatment. For example, should changes in an ISG such as CXCL1O be used to predict who is at risk for reactivating? It is also not clear whether the model in coinfection represents reactivation in HBV monoinfection.

Current HBV cure strategies focus on either eradicating all remnants of cccDNA from the liver or suppressing cccDNA transcription (i.e., a functional cure). We and others have reported evidence that cccDNA transcription may become suppressed (7, 25). It would be important to know if interferons were responsible for transcriptional silencing of cccDNA more generally, and whether reactivation in monoinfected people was due to diminished interferon levels. Indeed, it is notable that the clinical formulation of interferon, pegylated interferon $\alpha$, is the only FDA-approved medicine for HBV that leads to loss of HBsAg, although it does so infrequently. Thus, the Cheng et al. study (24), and HBV-HCV coinfection more generally, are important steps that raise deeper questions about HBV cure.

\section{Acknowledgments}

This work was supported by NIH grants R01 AI138810 (to AB and CT) and R01 AI116269 (to CT).

Address correspondence to: Ashwin Balagopal, Johns Hopkins University School of Medicine, Division of Infectious Diseases, 855 North Wolfe Street, Suite 535, Baltimore Maryland 21205, USA. Phone: 443.287.7666; Email: abalago1@jhmi.edu.

1. Ward JW, Hinman AR. What is needed to eliminate hepatitis B virus and hepatitis $C$ virus as global health threats. Gastroenterology. 2019;156(2):297-310.

2. Mahoney FJ. Update on diagnosis, management, and prevention of hepatitis B virus infection. Clin Microbiol Rev. 1999;12(2):351-366.

3. Spearman CW, Dusheiko GM, Hellard M, Sonderup M. Hepatitis C. Lancet. 2019;394(10207):1451-1466. 
4. Cacciola I, Pollicino T, Squadrito G, Cerenzia G, Orlando ME, Raimondo G. Occult hepatitis B virus infection in patients with chronic hepatitis C liver disease. N Engl J Med. 1999;341(1):22-26.

5. Kruse RL, et al. Clinical outcomes of hepatitis $B$ virus coinfection in a United States cohort of hepatitis $\mathrm{C}$ virus-infected patients. Hepatology. 2014;60(6):1871-1878

6. Oropeza CE, Tarnow G, Sridhar A, Taha TY, Shalaby RE, McLachlan A. The regulation of HBV transcription and replication. Adv Exp Med Biol. 2020;1179:39-69.

7. Balagopal A, et al. Single hepatocyte hepatitis B virus transcriptional landscape in HIV coinfection. J Infect Dis. 2020;221(9):1462-1469.

8. Lau GK, et al. Early is superior to deferred preemptive lamivudine therapy for hepatitis B patients undergoing chemotherapy. Gastroenterology. 2003;125(6):1742-1749.

9. Huang YH, et al. Randomized controlled trial of entecavir prophylaxis for rituximab-associated hepatitis $\mathrm{B}$ virus reactivation in patients with lymphoma and resolved hepatitis B. JClin Oncol. 2013;31(22):2765-2772.

10. Raimondo G, et al. Longitudinal evaluation reveals a complex spectrum of virological profiles in hepatitis $\mathrm{B}$ virus/hepatitis $\mathrm{C}$ virus-coinfected patients. Hepatology. 2006;43(1):100-107.

11. Nguyen LH, et al. Ethnic differences in viral dominance patterns in patients with hepatitis $\mathrm{B}$ virus and hepatitis $\mathrm{C}$ virus dual infection. Hepa- tology. 2011;53(6):1839-1845.

12. De Mitri MS, et al. Low replication and variability of $\mathrm{HBV}$ pre-core in concomitant infection with hepatitis B and hepatitis $\mathrm{C}$ viruses. Arch Virol. 2007;152(2):395-404.

13. Chen SY, et al. Mechanisms for inhibition of hepatitis $\mathrm{B}$ virus gene expression and replication by hepatitis $\mathrm{C}$ virus core protein. J Biol Chem. 2003;278(1):591-607.

14. Bellecave $P$, et al. Hepatitis $B$ and $C$ virus coinfection: a novel model system reveals the absence of direct viral interference. Hepatology. 2009;50(1):46-55.

15. Balagopal A, Thio CL. Editorial commentary: Another call to cure hepatitis B. Clin Infect Dis. 2015;61(8):1307-1309.

16. Wiegand SB, et al. Dominance of hepatitis C virus (HCV) is associated with lower quantitative hepatitis B surface antigen and higher serum interferon- $\gamma$-induced protein 10 levels in HBV/HCV-coinfected patients. Clin Microbiol Infect. 2015;21(7):710.e1-710.e9.

17. Suslov A, Boldanova T, Wang X, Wieland S, Heim MH. Hepatitis B virus does not interfere with innate immune responses in the human liver. Gastroenterology. 2018;154(6):1778-1790

18. Cheng X, et al. Hepatitis B virus evades innate immunity of hepatocytes but activates cytokine production by macrophages. Hepatology. 2017;66(6):1779-1793.

19. Thio CL, Netski DM, Myung J, Seaberg EC,
Thomas DL. Changes in hepatitis B virus DNA levels with acute HIV infection. Clin Infect Dis. 2004;38(7):1024-1029.

20. Collins JM, et al. Hepatitis B virus reactivation during successful treatment of hepatitis $C$ virus with sofosbuvir and simeprevir. Clin Infect Dis. 2015;61(8):1304-1306.

21. Bersoff-Matcha SJ, et al. Hepatitis B virus reactivation associated with direct-acting antiviral therapy for chronic hepatitis $\mathrm{C}$ virus: a review of cases reported to the U.S. Food and Drug Administration Adverse Event Reporting System. Ann Intern Med. 2017;166(11):792-798.

22. Mücke MM, et al. Hepatitis B virus reactivation during direct-acting antiviral therapy for hepatitis C: a systematic review and meta-analysis. Lancet Gastroenterol Hepatol. 2018;3(3):172-180.

23. Yeh ML, et al. Hepatitis B-related outcomes following direct-acting antiviral therapy in Taiwanese patients with chronic HBV/HCV co-infection [published online February 14, 2020]. J Hepatol. https://doi.org/10.1016/j.jhep.2020.01.027.

24. Cheng X, et al. Diminished hepatic IFN response following $\mathrm{HCV}$ clearance triggers $\mathrm{HBV}$ reactivation in coinfection. JClin Invest. 2020;130(6):3205-3220.

25. Zhang W, et al. PRMT5 restricts hepatitis B virus replication through epigenetic repression of covalently closed circular DNA transcription and interference with pregenomic RNA encapsidation. Hepatology. 2017;66(2):398-415. 